\title{
RELAÇÃO ENTRE ENXAQUECA, DIABETES MELLITUS E EXERCÍCIO FÍSICO
}

\author{
RELATION BETWEEN MIGRAINE, DIABETES MELLITUS AND PHYSICAL EXERCISE
}

Andy Petroianu; Ana Rita da Glória Soares²; Cibele Grossi Rocha³; Cristiane de Mattos Souza4; Florença de Aguiar Cardoso ${ }^{5}$ \& Marilene Lucinda Silva ${ }^{6}$

\begin{abstract}
'Docente do Departamento de Cirurgia da Faculdade de Medicina da UFMG; Docente Livre da Escola Paulista de Medicina - UNIFESP e da Faculdade de Medicina de Ribeirão Preto - USP; Doutor em Fisiologia e Farmacologia; Pesquisador IA do CNPq. ${ }^{2}$ Médica Residente de Clínica Médica. ${ }^{3}$ Bolsista da FAPEMIG; Médica Residente de Neurologia da Santa Casa de Misericórdia de Belo Horizonte. ${ }^{4}$ Médica ${ }^{5}$ Médica. ${ }^{6}$ Médica; Residente de Clínica Médica do Hospital Belo Horizonte.

Correspondência: Prof. Andy Petroianu. Avenida Afonso Pena, 1626 - Apto.1901 - CEP 30130-005 - Belo Horizonte, MG. Fone / FAX: (31) 274-7744
\end{abstract}

PETROIANU A; SOARES ARG; ROCHACG; SOUZA CM; CARDOSO FA \& SILVAML. Relação entre enxaqueca, diabetes mellitus e exercício físico. Medicina, Ribeirão Preto, 33: 515-519, out./dez. 2000.

RESUMO: A enxaqueca pode ocorrer em pessoas susceptíveis a vários fatores, incluindo o jejum e o exercício físico. De acordo com a literatura, esses fenômenos poderiam relacionar-se com os níveis de glicemia.

Objetivo: Pesquisar a relação entre o diabetes mellitus, a enxaqueca e o exercício físico.

Casuística e Método: Foram entrevistados 100 diabéticos (50 do tipo 1 e 50 do tipo 2) e 100 pessoas normais (grupo-controle) sem associação com enxaqueca e exercício físico.

Resultados: A enxaqueca ocorreu em $28 \%$ no grupo-controle. Ela esteve presente em $32 \%$ dos diabéticos tipo 1, sendo que um doente relatou melhora da cefaléia após o início do diabetes; outros $20 \%$ passaram a ter enxaqueca após o estabelecimento do diabetes; dois pacientes referiram piora após o início do diabetes. A enxaqueca esteve presente também em 18\% dos diabéticos tipo 2. Quanto à relação entre enxaqueca e exercício físico, observou-se que dos 38 pacientes diabéticos que praticavam esportes rotineiramente, apenas 20,5\% apresentaram enxaqueca, enquanto que, dos 60 diabéticos que não faziam exercício físico, $30 \%$ relataram esse quadro. No grupo-controle, os achados foram proporcionalmente os mesmos: das 71 pessoas normais com vida sedentária, 31\% apresentaram enxaqueca. Entre os 29 indivíduos que praticavam esporte regularmente a taxa de enxaqueca foi de $20,7 \%$.

Conclusão: A prática de exercício físico associou-se a menor freqüência de enxaqueca.

UNITERMOS: Enxaqueca. Diabetes Mellitus. Exercício.

\section{INTRODUÇÃO}

A enxaqueca caracteriza-se pelo aparecimento de cefaléia recorrente, em geral, com caráter pulsátil, precedida ou não de sintomas neurológicos focais, denominados de aura. Seguem-se, às vezes, náuseas, vômitos, foto e fonofobia. Alguns sintomas premonitórios podem aparecer horas ou dias antes da cefaléia, tais como: hiperatividade, depressão nervosa, irritabilidade, bocejos repetidos, dificuldade de memória, desejo por determinados alimentos, sonolência e outros. Essa afecção parece ocorrer em pessoas predispostas e é desencadeada por muitos fatores, entre eles a vasodilatação e o jejum. É possível que o jejum esteja relacionado a uma redução da glicemia, que seria o fator desencadeante, neste caso, da cefaléia. A rela- 
ção entre enxaqueca e glicemia é apontada pela literatura com uma incidência menor de enxaqueca entre os diabéticos ${ }^{(1,2)}$. Segundo Blau \& Pyke (1970), as crises de enxaqueca reduziram, em frequiência e intensidade, após o estabelecimento do diabetes ${ }^{(1,2)}$.

Em relação aos fenômenos vasculares, constatou-se que os diabéticos apresentam uma menor capacidade de vasodilatação cerebral, mesmo em presença de $\mathrm{CO}_{2}$, que é um potente vasodilatador cerebral $^{(2,3)}$. Talvez esse fato contribua, em parte, para a menor incidência de crises de enxaqueca. $\mathrm{O}$ mecanismo desse fenômeno vascular, de acordo com Dondona et al $(1985)^{(3)}$ seria mediado pela menor concentração de prostaciclina nos diabéticos ${ }^{(3)}$. Tal bloqueio à vasodilatação poderia decorrer da ação de ácidos graxos e outros lipídios que alteram a resposta do músculo liso vascular a substâncias vasoativas, inibem a secreção de prostaglandinas e aceleram sua degradação ${ }^{(3,4)}$. Por outro lado, a ativação plaquetária libera fatores plaquetários, que são substâncias vasoativas, diretamente implicadas na patogênese da enxaqueca e que aumentam em presença de diabetes. Nessa afecção também já foram detectados maiores níveis plasmáticos de tromboxane $\mathrm{A}^{(3,4)}$.

De acordo com a literatura, existe relação entre as crises de enxaqueca e o exercício físico, bem como entre a prática de esportes e diabetes ${ }^{(2,3,5)}$. Vários artigos relatam a existência da cefaléia benigna de esforço, de caráter pulsátil e curta duração que ocorre durante a prática de exercícios ${ }^{(5)}$. Sua etiopatogenia é ainda desconhecida, porém acredita-se que seja decorrente de uma hipoperfusão cerebral acompanhada de edema ${ }^{(5)}$. Além disso, segundo dados angiográficos, ocorre vasoespasmo assimétrico e difuso ${ }^{(6)}$.

Com relação à enxaqueca, há relatos de casos em que o exercício físico reduziu ou preveniu crises de enxaqueca e outros em que ele as desenca$\mathrm{deou}^{(7,8,9)}$. O gatilho, nesse caso, poderia estar relacionado a uma descarga alta de adrenalina, que é comprovada por sua excreção urinária aumentada ${ }^{(10)}$.

No que diz respeito ao diabetes, é indiscutível a inclusão da atividade física como complementação terapêutica ${ }^{(5,6,10)}$. Seus benefícios estão diretamente ligados ao aumento do rendimento cardiovascular, do tono e flexibilidade muscular e da produção de energia por meio de processos metabólicos e hormonais, além da diminuição do estresse. O exercício físico induz, ainda, a longo prazo, uma menor resistência periférica à insulina ${ }^{(11)}$.

Seguindo uma linha de pesquisa voltada ao diabetes mellitus, decidiu-se verificar a associação entre diabetes e enxaqueca e também esclarecer uma eventual influência do exercício físico nesse quadro.

\section{CASUÍSTICA E MÉTOdo}

Este estudo, em nível ambulatorial, foi conduzido em dois grupos.

Grupo 1: 100 diabéticos, sendo:

- 50 do tipo 1, congênita, (19 homens e 31 mulheres), com a idade variando entre 18 e 75 anos (Média $=35,6$ anos);

- 50 do tipo 2 ( 16 homens e 34 mulheres), com a idade entre 32 e 90 anos (Média = 58,8 anos). O tempo de doença foi muito variável, sendo que a maioria a descobriu por acaso, em exame de rotina ou em busca de diagnóstico para queixas diversas.

Grupo 2: controle - formado por 100 pessoas voluntárias (31 homens e 69 mulheres), com a idade variando entre 18 e 76 anos (Média $=36,6$ anos), sem dibetes mellitus ou qualquer doença aparente.

Realizou-se a pesquisa com base em um questionário composto pelos itens relacionados a seguir.

- Diabetes: classificação em tipo 1 ou tipo 2, uso de medicamentos para o controle dessa doença, regularidade com que foram seguidas as recomendações médicas (dieta e medicamentos), segundo os próprios relatos dos doentes;

- Enxaqueca: o presente trabalho seguiu o seguinte critério para a avaliação de enxaqueca: presença de cefaléia de forte intensidade, acompanhada de, pelo menos, duas das seguintes manifestações: náuseas, vômitos, alterações visuais, fotofobia, fonofobia, mal-estar e alterações do sono. O desencadeamento da cefaléia por algum tipo de medicamento, também foi pesquisado. Os critérios de melhora ou piora da enxaqueca foram verificados pelos relatos dos pacientes.

- Diabetes $x$ enxaqueca: perguntou-se a relação entre a enxaqueca e o diabetes quanto à melhora ou piora na duração, frequiência e/ou intensidade dos sintomas e a relação dessas mudanças com o tratamento.

Exercício físico: investigou-se a prática de esportes, data de início, frequiência, tipo de exercício, sua regularidade e intensidade, bem como sua relação com a enxaqueca.

Os resultados foram comparados pelo teste do qui quadrado. Foram consideradas significativas as diferenças superiores aos níveis de $\mathrm{P}<0,05$. 


\section{RESULTADOS}

A enxaqueca esteve presente em $28 \%$ dos pacientes do grupo-controle e em $25 \%$ dos diabéticos (32\% tipo 1 e $18 \%$ tipo 2). Em ambos os grupos, a enxaqueca predominou em mulheres (Tabela 1).

Entre os 16 diabéticos tipo 1 com enxaqueca, observou-se que:

- 10 pacientes iniciaram os episódios de enxaqueca após o diagnóstico do diabetes;

- dois tiveram aumento do número de episódios de enxaqueca, com piora de sua intensidade após o estabelecimento do diabetes;

- dois pacientes não souberam informar a relação da enxaqueca com o diabetes;

- um melhorou a enxaqueca após o diagnóstico do diabetes;

- um não apresentou alteração nos episódios de enxaqueca.

Entre os nove diabéticos tipo 2 com enxaqueca, observou-se:

- dois melhoraram a enxaqueca após o diagnóstico do diabetes;

- três constataram aumento do número de episódios de enxaqueca com piora de sua intensidade;

- três não perceberam mudanças nos episódios de enxaqueca com o advento da doença;

- um não soube informar a relação da enxaqueca com o diabetes.
Nos dois grupos de diabéticos, 14 pessoas seguiram corretamente as orientações médicas de dieta e medicação:

- quatro apresentaram piora dos episódios de enxaqueca após o início do tratamento do diabetes;

- oito iniciaram as crises após o diagnóstico e tratamento do diabetes;

- um apresentou melhora após o início do tratamento; - um não apresentou alteração.

Nove pessoas não seguiram rigorosamente as recomendações médicas:

- três apresentaram melhora das crises;

- com cinco não ocorreu alteração do quadro anterior;

- três relataram piora das crises após o início do tratamento.

Quanto à prática de esportes, 29 pessoas normais e 38 diabéticos exercitaram-se regularmente (Tabelas I e II). Essas tabelas mostram a freqüência de enxaqueca entre os que praticam esporte.

No grupo-controle a maior proporção dos que praticavam esporte era de homens $(45,2 \%$ contra $21,7 \%$ de mulheres). A caminhada foi o esporte mais praticado (16 pessoas), sendo que cinco delas apresentaram enxaqueca. Os outros esportes mais praticados foram: natação (6), musculação (3), corrida (4), ginástica (1), peteca (1) e ciclismo (1). Desses, somente um, que praticou musculação, tinha enxaqueca. Quanto às 71 pessoas que não praticavam esporte, 22 relataram a presença de enxaqueca.

Tabela I - Freqüência de enxaqueca e prática de esportes de acordo com o sexo em diabéticos e no grupo-controle de pessoas normais.

\begin{tabular}{lcccccc}
\hline & \multicolumn{2}{c}{ Controle $(100)$} & \multicolumn{2}{c}{ Diabetes } & Tipo 1 $(50)$ & \multicolumn{2}{c}{ Diabetes } & Tipo $2(50)$ \\
\cline { 2 - 7 } & $\begin{array}{c}\text { Masculino } \\
(31)\end{array}$ & $\begin{array}{c}\text { Feminino } \\
(69)\end{array}$ & $\begin{array}{c}\text { Masculino } \\
(19)\end{array}$ & $\begin{array}{c}\text { Feminino } \\
(31)\end{array}$ & $\begin{array}{c}\text { Masculino } \\
(16)\end{array}$ & $\begin{array}{c}\text { Feminino } \\
(34)\end{array}$ \\
\hline Enxaqueca & $6(19,3 \%)$ & $22(31,9 \%)$ & $3(17,6 \%)$ & $13(41,9 \%)$ & $1(6,2 \%)$ & $8(23,5 \%)$ \\
Prática de esportes & $14(45,2 \%)$ & $15(21,7 \%)$ & $8(47,1 \%)$ & $14(45,2 \%)$ & $3(18,7 \%)$ & $13(38,2 \%)$
\end{tabular}

Tabela II - Freqüência de enxaqueca de acordo com a realização de exercício físico em diabéticos e no grupo-controle de pessoas normais.

\begin{tabular}{|c|c|c|c|c|c|c|}
\hline \multicolumn{7}{|c|}{ Enxaqueca } \\
\hline \multirow{3}{*}{ Exercício físico } & \multirow{2}{*}{\multicolumn{2}{|c|}{ Controle (100) }} & \multicolumn{4}{|c|}{ Diabéticos (100) } \\
\hline & & & \multicolumn{2}{|c|}{ Tipo 1 (50) } & \multicolumn{2}{|c|}{ Tipo 2 (50) } \\
\hline & Sim & Não & Sim & Não & Sim & Não \\
\hline Sim & 6 & 23 & 7 & 15 & 0 & 16 \\
\hline Não & 22 & 49 & 9 & 9 & 9 & 25 \\
\hline
\end{tabular}


Entre os diabéticos tipo 1, não houve diferença entre a proporção de homens e mulheres que praticavam esporte. A caminhada também foi o exercício mais praticado: 20 doentes, dos quais sete relataram enxaqueca. Os outros esportes foram: futebol (3), natação (1), este com enxaqueca, luta(1) e vôlei (1), sendo que nenhum desses apresentou enxaqueca. Por outro lado, entre os diabéticos do tipo 2 a maior proporção dos que praticavam esporte foi de mulheres $(38,2 \%$ contra $18,7 \%$ de homens).16 praticaram caminhada e uma, ginástica. Nenhum deles queixava de enxaqueca.

Em resumo, quanto à relação entre enxaqueca e exercício físico, observou-se que dos 38 pacientes diabéticos que praticavam esportes rotineiramente, apenas $20,5 \%$ apresentaram enxaqueca, enquanto que, dos 60 diabéticos que não faziam exercício físico, $30 \%$ relataram esse quadro. No grupo-controle, os achados foram proporcionalmente os mesmos: das 71 pessoas normais com vida sedentária, $31 \%$ apresentaram enxaqueca. Entre os 29 indivíduos que praticavam esporte regularmente a taxa de enxaqueca foi de $20,7 \%$.

\section{DISCUSSÃO}

Este estudo mostrou uma frequência de enxaqueca semelhante entre as pessoas do grupo-controle e os diabéticos tipo 1. Já nos diabéticos tipo 2, a incidência de enxaqueca foi menor. Se forem comparados os dois grupos de diabéticos, observa-se que a grande maioria dos diabéticos tipo 1 pioraram a enxaqueca antes existente ou iniciaram os episódios de enxaqueca após o diagnóstico do diabetes. Já nos diabéticos tipo 2, em aproximadamente metade deles, a enxaqueca melhorou e na outra metade ela piorou. Observa-se, ainda, que a maioria dos pacientes que seguiam o tratamento, mantendo, presumivelmente, seus níveis glicêmicos próximos ao normal, referia piora das crises de enxaqueca ou revelava seu aparecimento. Já na maior parte dos pacientes que não seguiu as orientações médicas, mantendo provavelmente sua glicemia mais alta, as crises de enxaqueca melhoraram ou não apresentaram alteração do quadro.

Não se pode afirmar que a enxaqueca esteja diretamente relacionada ao nível de açúcar no sangue, mas sabe-se que a glicemia afeta o sistema nervoso autônomo, que, por sua vez, controla o tono vascu$\operatorname{lar}^{(5,6,11)}$. Como a enxaqueca é uma cefaléia de origem vascular, a glicemia poderia, eventualmente, influenciála $^{(1,2,11)}$. É muito importante ressaltar que as crises de enxaqueca reduzem com o avançar da idade, tanto em frequiência, quanto em intensidade. Portanto, é possível que esse fato tenha contribuído para a melho- ra, na clínica da enxaqueca, de alguns pacientes de nossa casuística. No presente trabalho, não foi realizada associação dos dados avaliados com a faixa etária.

Com relação ao exercício físico, é sabido que ele oferece inúmeros benefícios a quem o pratica. Dentre esses, estão a prevenção de distúrbios cardiovasculares e a redução da obesidade, que agravam o diabetes ${ }^{(7,9)}$. No entanto, considerando que foram selecionados apenas os pacientes que se enquadravam como portadores de enxaqueca, e eles não relataram crises durante o exercício, poder-se-ia pressupor que a prática de atividade física esteve relacionada a um alívio do quadro clínico. Segundo alguns autores, o exercício físico vigoroso seria um método eficaz de prevenir ou aliviar crises de enxaqueca. No presente trabalho, observou-se esse fato, já que as crises de enxaqueca foram mais comuns entre os praticantes de caminhada do que entre os que praticantes de esportes mais vigorosos como corrida, natação e ginástica, apesar de esse dado não ser estatisticamente significativo. Observou-se, ainda, que nenhum paciente diabético tipo 2, que realizou exercício, desenvolveu enxaqueca, levando a crer que, nesse grupo, mesmo exercícios mais leves, como a caminhada, seriam capazes de prevenir as crises.

\section{CONCLUSÕES}

Na presente pesquisa, a enxaqueca foi menos freqüente em diabéticos tipo 2 do que nos tipo 1 e nas pessoas normais. Exercícios físicos mais vigorosos associaram-se a menor presença de enxaqueca em diabéticos tipo 1 e pessoas normais. Nos diabéticos tipo 2, o exercício, mesmo leve, parece ser capaz de prevenir a enxaqueca. As mulheres apresentaram mais enxaqueca do que os homens, independente ou não da prática de exercício físico. Novas pesquisas em pacientes diabéticos e com enxaqueca são necessárias para entender melhor a influência da glicemia nesse quadro e esclarecer a relação entre prática de exercício físico com a glicemia e conseguinte enxaqueca.

\section{AGRADECIMENTOS}

Os autores agradecem ao Hospital Universitário São José, Ambulatório da Faculdade de Ciências Médicas de Minas Gerais, Hospital das Clínicas da UFMG, Santa Casa de Misericórdia, Instituto Mineiro de Nefrologia, todos em Belo Horizonte, além do Grupo de Diabéticos de Araxá pela cooperação em permitirem as entrevistas com os doentes.

Somos gratos também ao CNPq e à FAPEMIG pelo apoio financeiro. 
PETROIANU A; SOARES ARG; ROCHA CG; SOUZA CM; CARDOSO FA \& SILVA ML. Relation between migraine, diabetes mellitus and physical exercise. Medicina, Ribeirão Preto, 33: 515-519, oct./dec.2000.

ABSTRACT: Background - Migraine may be due to several factors, including the fast and physical exercise. According to literature, these phenomena may be related to glicemia.

Purpose - The present study verified the relation between diabetes mellitus, migraine and physical exercise.

Methods - This study assessed 100 diabetics (50 type 1 and 50 type 2) and 100 normal people (control group) in order to study the relation between diabetes mellitus, migraine and physical exercise.

Results - Migraine occurred in $28 \%$ of control group and $32 \%$ of diabetics type 1 : migraine was reduced in one patient after starting diabetes, $20 \%$ developed migraine in presence of diabetes and 2 patients worsen their migraine episodes after diabetes. Migraine was present in $18 \%$ of the diabetics type 2. $20,5 \%$ of diabetics who practiced exercises had migraine and $30 \%$ of sedentaries had such episodes. The results of control group were similar: $31 \%$ of 71 sedentaries had migraine; and $21,7 \%$ of 29 who regularly practice exercises had migraine.

Conclusions - Physical exercise is associated with less migraine either in control or diabetic groups.

UNITERMS: Migraine. Diabetes Mellitus. Exercise.

\section{REFERÊNCIAS BIBLIOGRÁFICAS}

1 - BLAU J N \& PYKE D A. Effect of diabetes on migraine. Lancet II: 241-243, 1970.

2 - BURN WK; MACHIN D \& WATERS WE. Prevalence of migraine in patients with diabetes. Br Med J 289: 1579-1580, 1984.

3 - DANDONA P; JAMES IM \& BECKETT AG. Prevalence of migraine in patients with diabetes. Br Med J 290: 467-468, 1985.

4 - ADAMS RD, VICTOR M \& ROPPER AH. Headaches and other craniofacial pains. In: ADAMS RD; VICTOR M \& ROPPER AH. Principles of neurology. 6th ed. Mc Graw Hill, New York, p. 167-193,1998.

5 - BASOGLU T; OZBENLI T; BERNAY I; SAHIN M; ONUR A; DEMIRCALI AE; COSKUN C \& ONEN T. Demonstration of frontal hypoperfusion in benign exertional headache by technetium-99m-HMPAO SPECT. J Nucl Med 37:1172-1174, 1996.

6 - SILBERT PL; HANKEY GJ; PRENTICE DA \& APSIMON HT. Angiographically demonstrated arterial spasm in a case of benign sexual headache and benign exertional headache. Aust N Z J Med 19: 466-468, 1989.
7 - VAN GJ. Relief of common migraine by exercise. J Neurol Neurosurg Psychiatry 50: 1700-1701, 1987.

8 - GARCIA AE; CABRERA F; TEJEIRO J; JIMENES JFJ \& VAQUERO A. Delayed postexercional headache, intracranial hypotencion and racket sports. J Neurol Neurosurg Psychiatry 40: 367-372, 1992.

9 - FERRARI MD. Relief of common migraine by exercise. J Neurol Neurosurg Psychiatry 51: 1011, 1988.

10 - STOICA E \& ENULESCU O. Catecholamine response to exercise in migrane. Rom J Neurol Psychiatry 32: 21-27, 1994.

11 - WASSERMAN DH \& ZINMAN B. Exercise in individuals with IDDM. Diabetes Care 17: 1732-1733, 1994.

Recebido para publicação em 08/06/2000

Aprovado para publicação em 30/11/2000 